\title{
Processing of tachistoscopic displays with controlled order of characters and spaces'
}

\author{
PETER SHAW 2,3 \\ STANFORD UNIVERSITY
}

The task used was designed to force Ss consistently to process the letters of a tachistoscopic display in the same spatial order, if Ss are able to process the letters in a brief display serially and if Ss have enough voluntary control over the selection of a processing order to use the order chosen by the $E$. In the main series of experiments the set of slides shown in each session included slides having a blank space in some position in the linear array of letters. For control, no-blank slides, a sharp, monotonic decrease in the percentage of correct detections of a signal letter with distance of the signal letter from the focusing dot was obtained. An unexpected result from the slides having a blank space was that detection of the signal letter was considerably more accurate on the slides having a blank space immediately following the signal letter than on the control slides having no blank space or the slides having a blank space immediately before the signal letter in the processing order. In one experiment blank spaces were replaced by black rectangles with no substantial change in the pattern of results. The results from the slides having a blank space or a black rectangle were interpreted as indicating that the onset of processing the image of a letter was determined by the distance in the display from the focusing dot to the letter and that the duration of processing an image of a letter was determined by the distance in the display from the letter to the next letter in the processing order.

The original purpose for the sequence of experiments to be reported was to provide evidence for or against the hypothesis that inputs to the brain from single, brief, two-dimensional, visual displays are processed in a spatially serial manner. More specifically, if a display of letters is presented to a human $S$ for so brief a duration that his eyes do not have time to move, then the spatially distributed letters are processed one at a time-rather than several simultaneously as in a camera. Sperling (1963) has presented data from a visual report task along with an interpretation in terms of serial scanning of a rapidly decaying visual information store. More recently, Sperling (1967) has presented other visual report data along with an interpretation in terms of parallel processing of a visual information store, followed by serial recoding of information into auditory storage. The choice between serial and simultaneous processing has been a central question in a series of visual detection experiments performed by Estes and his associates (Estes \& Taylor, 1964, 1966; Estes \& Wessel, 1966; Wolford, Wessel, \& Estes, 1968), and the assumption of serial processing is a crucial ingredient in the serial processing model proposed by Estes and Taylor (1964) and considered throughout the visual detection series.

Neither the experiments of Sperling nor the visual detection experiments have resolved the question of serial vs parallel processing. The detection technique appears to the writer to offer special advantages for investigating this question, but the latency evidence from the visual detection experiments has been inconclusive (Wolford, Wessel, \& Estes, 1968). The difficulty in this case may be that the variation in sensory processing time with stimulus variation is small compared to the variation in other components of the total response time. It is also true that a number of assumptions are needed to make inferences from observed latencies to alternative sensory processing mechanisms. Results from the response data of the detection task are complicated by two variables that will be discussed after the detection paradigm is described.

A typical trial in one of the experiments of Estes and his associates begins with the S's eyes fixated on a focusing dot in the center of a visual field. After a warning tone, a display of consonants filling a randomly selected subset of positions in a 4 by 4 array of potential positions is presented for a short interval (usually $50 \mathrm{msec}$ ) with the 4 by 4 array of potential positions centered on the position of the focusing dot. Each display contains one of two signal letters, e.g., either a $B$ or an $R$ but not both, in some randomly chosen position. The task of the $S$ is to indicate which critical letter appeared and to guess when unsure.

This paradigm has a desirable property for an experimental method to be used in investigating the question of serial or parallel processing in that the $\mathrm{S}$ must report only one letter. A one-letter report requires minimal usage of brain processes for retention over a period of many seconds, and, further, requires perception of an easily encoded part of the display that subtends a small visual angle. Thus, the visual detection task might be used to demonstrate a gradient of visual processing performance over an area of the display that is entirely within the region of good retinal sensitivity.

However, if processing the letters in a brief display is done serially, the visual detection task as employed in the past does not control two possibly important variables: the extent and the nature of the portion of the display processed before the critical letter. Work by Sperling (1960) has suggested that a brief display is initially stored in the nervous system in a representation that is characterized by rapid decay (the letters would be expected to become available in less than a second under the bright pre- and postexposure stimulus conditions of the present experiments), high information capacity, and preservation of the spatial relationships of the stimulus array. If the time to read out a letter from the initial, rapidly decaying, visual representation of a letter is an appreciable fraction of the total decay time, then the number of letters read out before the signal letter is reached must be an important determiner of the probability that the signal is still available to be read out of the decaying representation. If a blank space takes significantly less processing time than the same area filled with letters, then replacement of a letter by a blank space in the portion of the display processed before the signal should significantly facilitate detection of the signal-even if the blank space is separated from the signal by several noise letters.

The modified visual detection task used in the present experiments was designed with the purpose of controlling the S's processing order. If $\mathrm{Ss}$ are forced consistently to use the same processing order, then the size and the nature of the portions of the display processed before and after the signal are exactly known and can be systematically varied. In the modified visual detection paradigm, each trial begins with the S's eyes fixated on a focusing dot. After a warning click, a horizontal linear array of letters is flashed on the screen in a position immediately to the right of the position of the focusing dot. The task of the $S$ is to "read" the display from left to right, to indicate which of two 
alternative noise letters is the one in the first position to the right of the focusing dot, and to indicate which of the two signal letters is somewhere in that display. The exposure duration is adjusted for each $S$ so that he can be almost perfectly accurate on the noise letter in the first position if he begins the trial with his eyes fixated on the focusing dot or the first letter, but not otherwise.

If the letters in a brief display are processed serially, if the time to process a letter is sufficiently long, and if Ss can be instructed and constrained consistently to use the same processing order, then correct detection performance in the modified paradigm should be a monotonic, decreasing function of position of the signal in the stimulus array relative to the focusing dot. If, further, a blank space takes significantly less processing time than a similar area filled with letters, then a blank space before the signal in the S's processing order should facilitate detection of the signal and should facilitate detection more than a blank space following the signal. If, however, blank spaces before and after the signal should prove to exert equivalent effects on detection performance, a parallel, rather than serial, processing model would be suggested.

\section{EXPERIMENTS}

Common aspects of all five of the experiments to be reported were the apparatus, the warning click that preceded the stimulus exposure by $.5 \mathrm{sec}$, the bright pre- and postexposure field with a black focusing dot, and the brightness determinations for the preand postexposure field and for the stimulus field.

The displays for these experiments were generated by photographing white letters on a black velvet background with 35-mm, high-contrast, black-and-white copy film and then mounting the negatives as slides. The white cardboard letters photographed were block, capital letters, 11/4 in. high, made by Duro Co. The slides were seen as black letters on a bright background. The control displays contained 10 letters in a linear array that subtended $4 \mathrm{deg} 17 \mathrm{~min}$, i.e., $1.43 \mathrm{in}$. at a distance of $15.5 \mathrm{in}$. The critical letter appeared only in the first six positions, which together subtended $2 \mathrm{deg} 10 \mathrm{~min}$. The spaces between letters were the width of a letter. The focusing dot had a diameter about half the width of a letter and was seen, except when the display was presented, at the between-letters distance to the left of the point where the left-most letter appeared when presented.

All sessions began with a 4-min adaptation period in the experimental room, followed by 10 warm-up trials. There was a 3-min rest period in the middle of each session. For the data-collection sessions, Ss were run twice a day with the two sessions always at least an hour apart. For each experiment all slides appeared equally often in a session and all were equally likely to be presented on any trial.

The projectors of the automated, two-field, dual tachistoscope used in these experiments were located in a wooden box, $5 \mathrm{ft} 7 \mathrm{in.} \times 4 \mathrm{ft} 1 \mathrm{in}$. $x 2 \mathrm{ft} 4 \mathrm{in}$. high, standing on four 8 -in. legs on a table in a soundproofed, air-conditioned room. At each of the ends of the box was a subject station formed by a recess that was $8 \mathrm{in}$. wide, $15 \mathrm{in}$. deep, and the full height of the box. In this recess was a panel containing a ground-glass, rear-projection screen, 8 in. wide and $63 / 4$ in. high, centered vertically. Behind this screen was a black metal plate with a large circular aperture through which the displays were projected onto the screen to form an illuminated circle 2.06 in. in diam. A plastic eyepiece was mounted flush with the outer face of the box, 15 in. in front of the screen and aligned in height with the circular aperture. Below each observation station was suspended at lap height a response panel, 12 in. $x 10$ in., on which were a vertical and a horizontal array of four adjacent response buttons, each $1 \times .85 \mathrm{in}$.

Stimuli were projected by a random-access slide projector
(Spindler \& Sauppe Model SLX-750) modified to mount a special light source (Sylvania electronic tube No. R1131) characterized by rise time and decay time within $.05 \mathrm{msec}$. A second projector, optically identical to the first but holding a single slide that was clear except for the focusing dot, illuminated the screen between stimulus exposures. Brightness determinations made with a Macbeth Illuminometer for the stimulus field and the secondary (pre- and postexposure) field were 2.3 and $3.6 \mathrm{ft}-\mathrm{c}$, respectively, for Subject Station 1, and 1.6 and $1.4 \mathrm{ft}-\mathrm{c}$, respectively, for Station 2.

The control system of the tachistoscope was located in an adjacent room, visually and acoustically isolated from the display room. Each subject station had an intercom unit connected with a master unit in the control room.

\section{Experiment 1}

The primary purposes of Experiment 1 were to obtain stable estimates for several $S s$ of the function relating correct detection performance to position of the signal in the S's processing order and to obtain the pattern of facilitative effects of a blank space in various positions relative to the position of the signal in the processing order. It was expected that a blank space adjacent to the signal on either side would produce an extra increment in detection probability, because with these positions of the space only one noise letter is immediately adjacent to the signal and, therefore, only one noise letter is likely to interfere with the processing of the signal.

\section{Subjects}

Two Stanford graduate students and one Stanford undergraduate served as Ss and were paid for their participation. S GA had been in a preliminary experiment. No $S$ wore contact lenses.

\section{Stimulus Materials}

The 64 slides of Experiment 1 are listed in Table 1. The critical letter appeared in Positions 2 through 6 . The first letter in any array was either $\mathrm{A}$ or $\mathrm{N}$; other background letters were either $\mathrm{H}$ or $\mathrm{N} ;{ }^{4}$ the critical letter was either $\mathrm{B}$ or $\mathrm{R}$. For each position of the critical letter, a permutation of $\mathrm{Hs}$ and Ns was arbitrarily picked for the background; for each position of the critical letter and for each critical letter, there were two displays-the chosen permutation and one generated by replacing all $\mathrm{Hs}$ by Ns and all $\mathrm{Ns}$ by $\mathrm{Hs}$, and by changing any initial $\mathrm{H}$ to a rectangularly shaped A. The blank spaces in the arrays were made by taking out two or three background letters from a control display ready for photographing-two letters for Slide Types 4, 5, and 6 in Table 1, three letters for Types 8,9,10, and 12-16. Of the slides with a blank space, the two types with no letters between the blank space and the critical letter were of primary interest. The other slide types with a blank space were included to test for various effects that were expected to be smaller-for example, the effect of a blank space one letter away from the critical letter.

\section{Procedure}

Ss participated in 5 sessions of practice and 20 sessions of data collection. Ss AM and LF were run on Stations 1 and 2, respectively, simultaneously when it was possible; GA was always alone in the experimental room at Station 1. Each session had 256 E-paced, 9-sec, data-collection trials. The focusing dot was continuously present near the left edge of the illuminated circle except during stimulus presentation and during the 1 -msec dark period between trials. The linear stimulus array appeared at the same height as the dot and at the between-letters distance to the right of the focusing dot. A warning click was sounded through the intercom units $.5 \mathrm{sec}$ before the stimulus presentation.

During the practice sessions the stimulus duration for each $S$ 
Table 1

Slides for Experiment 1

\begin{tabular}{|c|c|c|c|c|c|c|}
\hline 1 & \multicolumn{2}{|c|}{$\begin{array}{l}\text { ABNNHHNNNH } \\
\text { ARNNHHNNNH } \\
\text { NB HHNNHHHN } \\
\text { NRHHNNHHHN }\end{array}$} & 9 & $\begin{array}{l}\text { AN } \\
\text { AN } \\
\text { NH } \\
\text { NH }\end{array}$ & $\begin{array}{l}\text { INB } \\
\text { INR } \\
\text { IHB } \\
\text { IHR }\end{array}$ & $\begin{array}{l}\text { HN } \\
\text { HN } \\
\text { NH } \\
\text { NH }\end{array}$ \\
\hline 2 & \multicolumn{2}{|c|}{$\begin{array}{l}\text { ANB HNNHHHN } \\
\text { ANRHNNHHHN } \\
\text { NHB NHHNNNH } \\
\text { NHRNHHNNNH }\end{array}$} & 10 & \multicolumn{3}{|c|}{$\begin{array}{l}\text { ABNNHHN } \\
\text { ARNNHHN } \\
\text { NBHHNNH } \\
\text { NRHHNNH }\end{array}$} \\
\hline 3 & \multicolumn{2}{|c|}{$\begin{array}{l}\text { AHNB NNHHNN } \\
\text { AHNRNNHHNN } \\
\text { NNHBHHNNHH } \\
\text { NNHRHHNNHH }\end{array}$} & 11 & \multicolumn{3}{|c|}{$\begin{array}{l}\text { AHNNHB NHNH } \\
\text { AHNNHRNHNH } \\
\text { NNHHNB HNHN } \\
\text { NNHHNRHNHN }\end{array}$} \\
\hline 4 & $\begin{array}{ll}\mathbf{A} & \mathbf{B I} \\
\mathbf{A} & \mathbf{R I} \\
\mathbf{N} & \mathbf{B H} \\
\mathbf{N} & \mathbf{R H}\end{array}$ & $\begin{array}{l}\text { NHHNN } \\
\text { NHHNN } \\
\text { HNNHH } \\
\text { HNNHH }\end{array}$ & 12 & \multicolumn{3}{|c|}{$\begin{array}{l}\text { ANBHNHN } \\
\text { ANRHNHN } \\
\text { NHBNHNH } \\
\text { NHRNHNH }\end{array}$} \\
\hline 5 & \multicolumn{2}{|l|}{$\begin{array}{l}\text { AHNB } \\
\text { AHNR } \\
\text { NNHB } \\
\text { NNHR }\end{array}$} & 13 & $\begin{array}{l}\mathbf{A} \\
\mathbf{A} \\
\mathbf{N} \\
\mathbf{N}\end{array}$ & \multicolumn{2}{|c|}{$\begin{array}{l}\text { HBNHNH } \\
\text { HRNHNH } \\
\text { NBHNHN } \\
\text { NRHNHN }\end{array}$} \\
\hline 6 & \multicolumn{2}{|c|}{$\begin{array}{l}\text { ABNNHHNN } \\
\text { ARNNHHNN } \\
\text { NBHHNNHH } \\
\text { NRHHNNHH }\end{array}$} & 14 & $\begin{array}{l}\text { AH } \\
\text { AH } \\
\text { NN } \\
\text { NN }\end{array}$ & \multicolumn{2}{|c|}{$\begin{array}{l}\text { BNHNH } \\
\text { RNHNH } \\
\text { B HNHN } \\
\text { RHNHN }\end{array}$} \\
\hline 7 & \multicolumn{2}{|c|}{$\begin{array}{l}\text { ANHNB NNHHN } \\
\text { ANHNRNNHHN } \\
\text { NHNHB HHNNH } \\
\text { NHNHRHHNNH }\end{array}$} & 15 & \multicolumn{2}{|c|}{$\begin{array}{l}\text { AHNNHB } \\
\text { AHNNHR } \\
\text { NNHHNB } \\
\text { NNHHNR }\end{array}$} & $\begin{array}{l}\mathbf{H} \\
\mathbf{H} \\
\mathbf{N} \\
\mathbf{N}\end{array}$ \\
\hline 8 & $\begin{array}{ll}\mathbf{A} & \mathbf{B} \\
\mathbf{A} & \mathbf{R} \\
\mathbf{N} & \mathbf{B} \\
\mathbf{N} & \mathbf{R} \\
\end{array}$ & $\begin{array}{l}\text { JNHHN } \\
\text { WNHHN } \\
\text { IHNNH } \\
\text { IHNNH } \\
\end{array}$ & 16 & \multicolumn{3}{|c|}{$\begin{array}{l}\text { AHNNHBN } \\
\text { AHNNHRN } \\
\text { NNHHNB H } \\
\text { NNHHNRH } \\
\end{array}$} \\
\hline
\end{tabular}

was decreased from $1 \mathrm{sec}$ to the final stimulus duration for that $S$; this data-collection duration was chosen on the basis of the practice data and was intended to give each $S$ a proportion correct of about .55 to .60 on his detection responses on the control slides that had no blank space and the critical letter in the sixth position. This procedure produced data-collection stimulus durations of $40 \mathrm{msec}, 20 \mathrm{msec}$, and $20 \mathrm{msec}$ for Ss GA, AM, and LF, respectively.

Neither latencies nor confidence judgments were taken. The top two buttons of the vertical array of response buttons were used for the verification response at each subject station with the upper button for the A response. At Station 1 the rightmost pair of the horizontal buttons was used for the detection response, with the S's right forefinger on the $B$ button and his right second finger on the $\mathrm{R}$ button.

The instructions to the Ss were as follows:

(1) Each display has a B or an $R$ in it, but not both, in a position other than first in the line. Push the $B$ button if you think the display has a $B$ in it and the $R$ button if you think it has an $R$; guess when unsure.

(2) There is a warning click $.5 \mathrm{sec}$ before each flash of a display.

(3) At the time of the flash of the display, have your eyes focused on the black dot near the left edge of the illuminated circle.

(4) Process the display from left to right: "read" the display from left to right.

(5) Push the A button if the leftmost letter in the display in an $\mathbf{A}$ and the $\mathbf{N}$ button if it is an $\mathrm{N}$. Then make your $B-R$ detection response.

(6) If it seems that your B or $R$ response is going to be slower than $3 \mathrm{sec}$, do not make it; if you do, your responses on the next trial will not be recorded.

(7) If you inadvertently push the wrong button for your
A-N verification response, do not make your B-R detection response for that trial. ${ }^{5}$

(8) It is important that you are always almost right as to whether the first letter is an A or $\mathbf{N}$ except for the trials on which the first letter is displaced to the right from the focusing dot; failures on these special trials are allowed in the data analysis.

(9) Try to be sure your face is touching the plastic eyepiece when the warning click is sounded.

(10) There is a few minutes break halfway through the session.

\section{Results}

Error proportions on the first-letter verifications were $.01, .03$, and .05 for Ss GA, AM, and LF, respectively. Verification errors were not counted for slide types having a blank space in the leftmost portion of the array (Slide Types 6, 10, and 12 in Table 1). For each $S$ there were approximately 4,000 properly recorded trials in which the first letter in the display was immediately to the right of the focusing dot. The practice-session trials, warm-up trials, and recording-equipment-failure triais were not included in any analyses; verification-error trials were not included in correct detection proportions.

The observed proportions of correct detection responses for each position of the critical letter and for each slide type are presented by $S$ in Figs. 1-3. The three curves in each figure represent the proportions for the control slides, with no blank space; the proportions for the postblank-zero slides, with a blank space immediately to the right of the critical letter; and the proportions for the preblank-zero slides, with the blank space immediately to the left of the critical letter. The other proportions are for the preblank-one and the postblank-one slides, with the blank space one letter to the left and right, respectively, from the critical letter.

Surprisingly, a blank space immediately following the critical letter in the processing order had a large, positive effect on detection performance, and a blank space immediately before the critical letter seems not to have had any positive effect on detection performance. Except for one point for S AM, all three curves for all three Ss are strictly monotonically decreasing from left to right.

To provide at least a rough assessment of the reliability of the observed effects, the proportion correct for each slide type having a blank space was compared with its control proportion-the proportion for the control slide type with the critical letter in the same position-by means of the statistic

$$
z=\frac{P_{1}-P_{2}}{\sqrt{P Q\left(\frac{1}{N_{1}}+\frac{1}{N_{2}}\right)}},
$$

where $N_{1}$ and $N_{2}$ are the numbers of observations in the two proportions, and $P$ and $Q$ are the proportions of correct and incorrect detections, respectively, for the combined sample. The value of $z$ may be interpreted as a deviate of the unit normal curve if $N_{1}$ and $N_{2}$ are reasonably large and if $P$ is neither very large nor very small. Ferguson (1958) suggests the rule of thumb that if $\min (P, Q) \times \min \left(N_{1}, N_{2}\right)$ is greater than five, then $z$ may be considered normal.

With respect to this criterion, a two-tailed $z$ test for comparison of each blank-space proportion with its control proportion is reasonable: the number of observations for each of these proportions is about 300 , and $\min (P, Q)$ has a range from .05 to .49 ; thus, $\min \left(\mathrm{N}_{1}, \mathrm{~N}_{2}\right) \times \min (\mathrm{P}, \mathrm{Q})$ has a range of 15 to 147. The results of these comparisons are as follows: each 


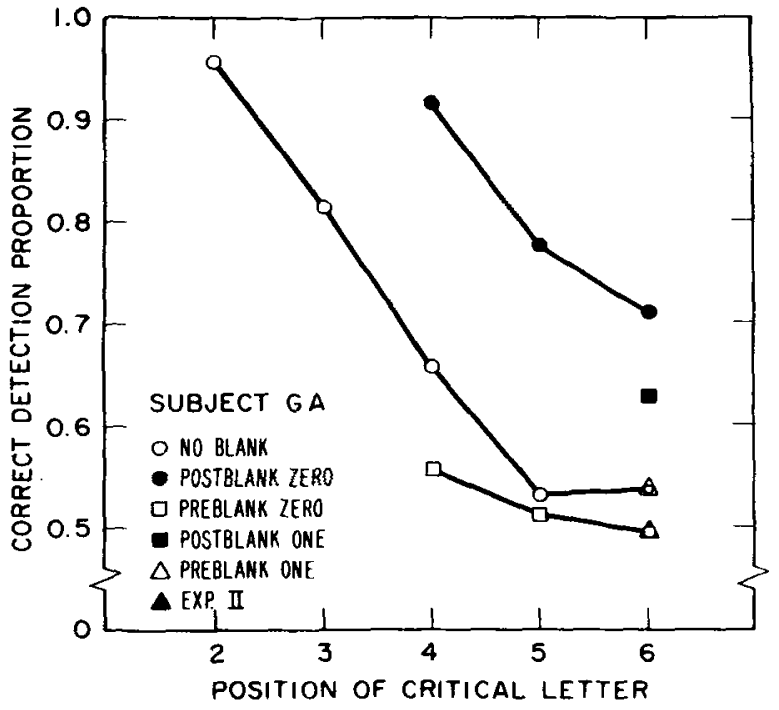

Fig. 1. Corsect-detection proportion as a function of position of the critical letter for $S$ GA.

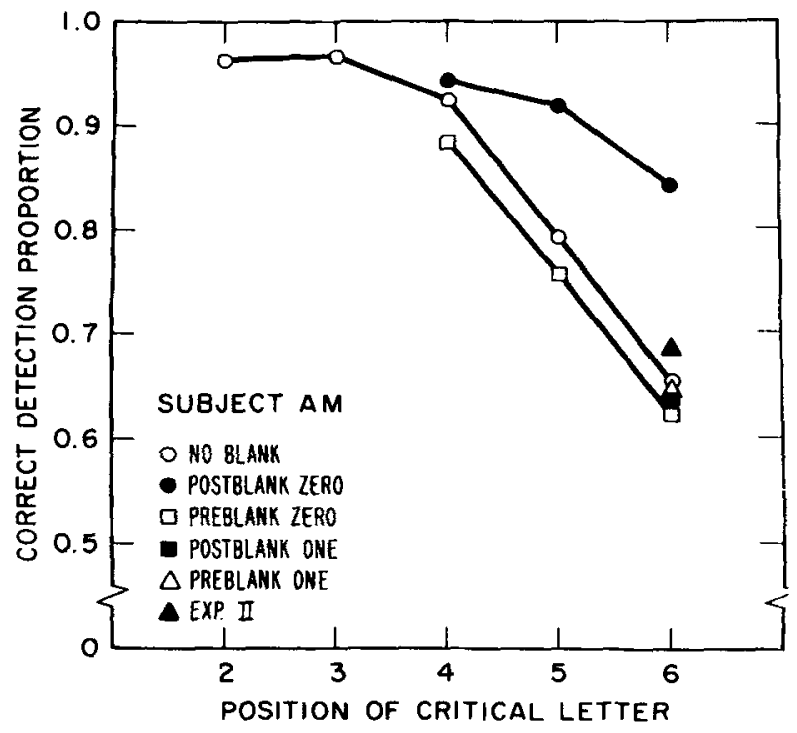

Fig. 2. Correct-detection proportion as a function of position of the critical letter for $\mathbf{S} A M$.

comparison of a postblank-zero proportion with its control proportion is significant at the .001 level with the exception of the comparison for Position 4 of S AM, which is not significant at the .05 level. None of the preblank-zero proportions are significantly different at the .05 level from their control proportions except for Position 4 of SGA. The detection proportions for the slide types with the first letter of the display shifted two or three positions from the focusing dot (Slide Types 6,10 , and 12 of Table 1) and the control proportions for the same positions are shown in Table 2. Only for Position 4 of S AM was the shifted proportion significantly different, at the .05 level, from the control proportion.

It is hard to doubt that a blank space immediately following the critical letter improved detection performance in this experiment. The smallness of the observed increment in detection performance produced by the postblank-zero space for Position 4

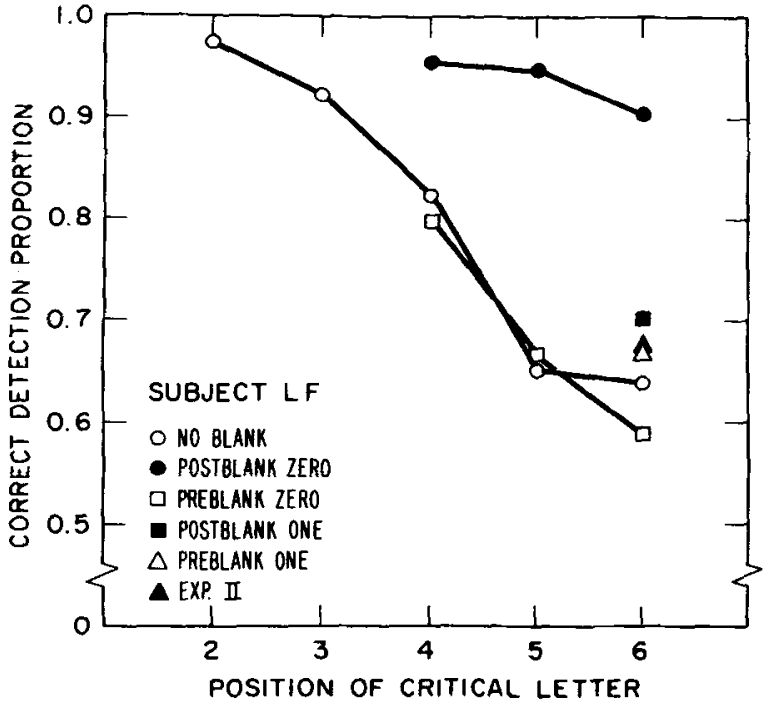

Fig. 3. Correct-detection proportion as a function of position of the critical letter for S LF.

of S AM is not important considering his already high level of performance on Position 4 control slides (93\%); the three comparisons significant at the .05 level among the remaining 24 comparisons show no pattern. In this experiment the effect of a blank space not immediately following the critical letter seems approximately equal to that of a space of the same length filled with background letters.

Two-tailed Wilcoxon sign tests were performed on each S's data to test whether detection performance on the first 10 sessions was different from the second 10 sessions and whether the first daily session was different from the second. To compare the first 10 sessions with the second 10 sessions, the correct-detection proportions for the 16 slide types for each $\mathrm{S}$ were computed for the first 10 sessions and for the second 10 sessions and regarded as 16 matched pairs. Similarly, 16 matched pairs were computed for a comparison of the initial daily sessions with the final daily sessions. Two of these tests were significant: AM's correct-detection performance was higher on his second 10 sessions than on his first $(p<.01)$; LF's initial daily sessions were more accurate than her final sessions $(p<.05)$.

\section{Discussion}

The decrease in detection performance as a function of angle from the focusing dot seems too large to be attributed to a decline in retinal sensitivity with visual angle. In particular, S GA is at chance performance on control slides for Position 6 where the visual angle from the focusing dot is only about $2 \mathrm{deg}$. The

Table 2

Detection Proportions for the Shifted Slide Types and the Control Slide Types of the Same Positions

\begin{tabular}{|c|c|c|c|c|}
\hline & & \multicolumn{3}{|c|}{ Position } \\
\hline & & 4 & 5 & 6 \\
\hline GA & $\begin{array}{l}\text { Shifted } \\
\text { Control }\end{array}$ & $\begin{array}{l}.644 \\
.658\end{array}$ & $\begin{array}{l}.529 \\
.531\end{array}$ & $\begin{array}{l}.497 \\
.537\end{array}$ \\
\hline $\mathbf{A M}$ & $\begin{array}{l}\text { Shifted } \\
\text { Control }\end{array}$ & $\begin{array}{l}.854 \\
.925\end{array}$ & $\begin{array}{l}.783 \\
.791\end{array}$ & $\begin{array}{l}.700 \\
.651\end{array}$ \\
\hline LF & $\begin{array}{l}\text { Shifted } \\
\text { Control }\end{array}$ & $\begin{array}{l}.799 \\
.822\end{array}$ & $\begin{array}{l}.704 \\
.651\end{array}$ & $\begin{array}{l}.629 \\
.639 \\
\end{array}$ \\
\hline
\end{tabular}


fact that preblank-zero proportions are not greater than their control proportions strongly suggests that a blank space on either side of the signal does not increase retinal sensitivity over its level for the corresponding control slides. In this case the great superiority of postblank-zero performance over control performance is not caused by a difference in retinal sensitivity to the critical letter in the two conditions. But, if retinal sensitivity is the same for the postblank-zero slides and control slides, then the decrement from unity for a given postblank-zero proportion is an upper bound on the extent to which the decrement from unity of the corresponding control proportion can be attributed to imperfect retinal sensitivity. For all three Ss the decrements from unity of the postblank-zero proportions for Positions 5 and 6 were much less than the decrements for Control Positions 5 and 6; and, therefore, the sharp monotonic decrease in control detection performance is not mainly caused by a loss of retinal sensitivity as a function of position of the critical letter.

While the type of spatial detection gradient expected on the basis of the serial processing considerations discussed above was obtained, a blank space before the signal did not facilitate detection performance. In the framework of serial processing of a decaying image, the result that a blank space before the signal does not facilitate detection suggests that a blank space does not take significantly less processing time than the same area filled with noise letters.

Four possible explanations for the great inequality between the effect of a blank space immediately before the signal and the effect of a blank space immediately after the signal were tested in Experiments 2-4.

\section{Experiment 2}

Experiment 2 is pertinent to the hypothesis that a blank space immediately following the signal letter serives as a "locator" and enables the processing mechanism to skip directly from the verification letter to the signal. The specific assumptions under examination were the following: (a) Letters are serially read out of a rapidly decaying visual representation, and this read-out is done in the instructed processing order. (b) The time to read out a single letter is a significant fraction of the average time that the amount of information in the representation of a letter is above a detection threshold. (c) The read-out jumps from the representation of the leftmost letter in a display to the representation of a letter immediately preceding a blank space in the instructed processing order.

\section{Subjects}

The same Ss participated as in Experiment 1.

\section{Stimulus Materials}

The stimuli were the four slides of Slide Type 2 of Table 1, i.e., the control slides for Position 6. Another identical set of four slides was shown on half the trials; the reason for having eight slides instead of four was to be sure that noises from the slide projector would not be clues to the identity of the slide readied for projection.

\section{Procedure}

This experiment was conducted on the day after Experiment 1 ended. There were two sessions, with 30 warm-up trials each rather than the usual 10 warm-up trials. The only other difference between Experiments 1 and 2 was in the instructions. The Ss were told that their task was still the same, that now the critical letter would always appear in Position 6, that there would be no blank spaces, and that they should maintain their near-perfect performance on verification of the leftmost letter.

\section{Results}

Verification error proportions were $.00, .01$, and .07 for Ss GA, AM, and LF, respectively. Correct-detection proportions for the first and second sessions were $(.50, .49),(.68, .70)$, and $(.68$, .67) for Ss GA, AM, and LF, respectively. Correct-detection proportions for both sessions combined appear in Figs. 1-3 as the triangles labeled "Exp. II" (with about 500 observations per S). The $\mathrm{z}$ tests computed to compare for each $\mathrm{S}$ the correct-detection proportion on Position 6, control slides in Experiment 1 with the two-session detection proportion in Experiment 2 were highly significant for Ss AM and LF. GA was at the chance detection level on these slides in both experiments.

\section{Discussion}

The proportions for the first and second sessions show no improvement in detection performance during this experiment. Any change from Experiment 1 in the read-out procedure used by the Ss was either of small consequence for detection performance or was accomplished mainly during the 30 warm-up trials of the first session.

While AM and LF were significantly more accurate when they were told that the critical letter would always appear in Position 6, the increment in detection performance was for all Ss less than a fourth that produced by a blank space immediately following a critical letter in Position 6. For this reason it seems unlikely that a scanning jump that is under voluntary control by the $\mathrm{S}$ can give an adequate account of the large facilitation of detection produced by a postblank-zero space. An ad hoc explanation assuming a scanning mechanism that is programmed for an automatic scanning jump to the letter preceding a blank space is not disconfirmed by the present experiment. (But this hypothesis does not by itself imply that detection on postblank-zero slides should be a monotonically increasing function of the length of the blank space. Preliminary results from an experiment in progress indicate, for example, that a postblank-zero slide with a blank space the width of three letters produces a significantly less accurate detection performance than a slide with the signal in the same position and with only blank space following the signal letter.)

\section{Experiment 3}

Experiment 3 was designed to test a pleasingly simple explanation of the result that the effect of a blank space before the critical letter is unequal to the effect of a blank space after the critical letter. Perhaps the large facilitation of detection by a postblank-zero space is related to a special property of the right halves of the asymmetrical critical letters, $B$ and $R$. This would be the situation, for example, if the distinguishing features of $B$ and $R$ are on the right side and if a blank space greatly facilitates performance on only the nearest features.

In contrast to Experiment 1 , this experiment used symmetrical critical letters, $\phi$ and $\theta$, which appeared only in Position 6. If symmetrical critical letters were also to show an asymmetrical effect of a blank space, then the results of Experiment 1 could not be attributed to the left-right asymmetry of $B$ and $R$. Experiment 2 had shown that, even if the position of the signal were known in advance of a trial, performance on a signal in Position 6 would still be poor enough to permit facilitation by a postblank-zero space.

Subjects

The same Ss participated as in the previous experiments.

\section{Stimulus Materials}

The stimuli were the control, preblank-zero, postblank-zero, preblank-one, and postblank-one slide types for Position 6 of 
Table 3

Correct Detection Proportions

\begin{tabular}{llll}
\hline & GA & AM & LF \\
\hline Postblank zero & .632 & .800 & .733 \\
Preblank zero & .493 & .640 & .663 \\
Control & .480 & .561 & .528 \\
Postblank one & .458 & .668 & .566 \\
Preblank one & .477 & .620 & .559 \\
\hline
\end{tabular}

Experiment 1 (Slide Types 11, 13, 14, 15, and 16 of Table 1) with the differences that all the background $\mathrm{Hs}$ were changed to rectangularly shaped $A s$, and the critical letters $B$ and $R$ were changed to $\theta$ and $\phi$, respectively.

\section{Procedure}

The 260 trials per session and the two sessions per day had all the same characteristics as the trials and sessions of Experiment 1. There were two practice sessions and six data-collection sessions. Ss were told that the critical letters had been changed to $\theta$ and $\phi$, that they should push the former $B$ response button when they detected or guessed $\theta$ and the former $R$ button when they detected or guessed $\phi$, that the critical letter would always appear in Position 6, and that everything else was the same as in Experiment 1.

\section{Results}

Verification error proportions were $.005, .02$, and .04 for Ss GA, AM, and LF, respectively. The correct-detection proportions for the different slide types for each $S$ are shown in Table 3 . Although the Ss had the small advantage of knowing that the critical letter would always appear in Position 6, the general level of correct-detection performance was somewhat lower with $\theta$ and $\phi$ as critical letters. For each $S, z$ tests were computed to compare each slide type with a blank space with its control slide type. For all three $\mathrm{Ss}$ the postblank-zero slide type was significantly different from the control slide type at the .001 level. For GA the other slide types were not significantly different at the .05 level from the control slide type; for AM the preblank-one slide type was not significantly different at the .05 level from the control slide type, but the preblank-zero and postblank-zero slide types did reach the .05 significance level; for LF the preblank-one and postblank-one slide types were not significantly different at the .05 level from the control slide type, but the preblank-zero slide type was.

\section{Discussion}

In summary, with $\theta$ and $\phi$ as critical letters, blank spaces produced approximately the same asymmetrical pattern of facilitation as found with $B$ and $R$ in Experiment 1. Although for two Ss the preblank-zero comparison was significant, the preblank-zero effect for these Ss was considerably less than the postblank-zero effect. The smallness of the preblank-zero effect with $\theta$ and $\phi$ will be given stronger support by the results of Experiment 5.

In Experiment 3 as in Experiment 2 the $\mathrm{Ss}$ knew in advance where the signal would be located. The postblank-zero facilitation found in Experiment 3 is, therefore, in addition to any processing advantages made possible by foreknowledge of the position of the signal.

\section{Experiment 4}

Masking seems an obvious interpretation of the facilitation of detection of a signal by an adjacent blank space. There must be a degree of proximity such that a noise letter is close enough to the signal to increase the difficulty of accurately identifying the contours of the signal. This difficulty may exist because, for example, parts of the signal letter and a close noise letter are in
Table 4

Correct Detection Proportions

\begin{tabular}{llll}
\hline & GA & AM & LF \\
\hline Postrectangle zero & .712 & .978 & .849 \\
Prerectangle zero & .501 & .789 & .768 \\
Control & .479 & .756 & .626 \\
Postrectangle one & .554 & .775 & .748 \\
Prerectangle one & .532 & .715 & .695 \\
\hline
\end{tabular}

the same receptor field of some neuron. A special problem of an explanation of this sort is the great disparity between the effect of a blank space before the signal and the effect of a blank space after the signal. To handle this problem a masking explanation must assume that an adjacent noise letter on the side of the signal away from the focusing dot has a much greater masking effect than on the side nearest the focusing dot. To test this type of explanation the blank spaces of the previous experiments were replaced in Experiment 4 by solid, black rectangles. The assumption that a black rectangle adjacent to the signal has a masking effect approximately equivalent to that of a noise letter implies that in Experiment 4 the correct-detection proportion on a postrectangle-zero slide (a slide having a rectangle coming immediately after the signal in the S's processing order) should be approximately the same as on a control slide with the signal in the same position and should be approximately the same as on a corresponding prerectangle-zero slide (a slide having a rectangle coming immediately before the signal).

Subjects

The Ss were the same three as in the previous experiments.

\section{Stimulus Materials}

The slides were the same as in Experiment 3 except that $B$ and $\mathrm{R}$ replaced $\theta$ and $\phi$, respectively, as critical letters, and each blank space of Experiment 3 was replaced by a solid, black rectangle. The blank space slides of Experiment 3 had been made by removing three letters from a complete display of cardboard letters prepared for the photographing of a control slide; each black-rectangle slide of the present experiment was made by replacing the appropriate three cardboard letters with a cardboard rectangle the height of a letter and the width of the space in a slide of any three adjacent letters.

\section{Procedure}

The 260 trials per session and the two sessions per day had all the same characteristics as the trials and sessions of Experiments 1 and 3 . There were two practice and eight data-collection sessions. Ss were told that black rectangles had replaced blank spaces, that $\mathrm{B}$ and $\mathrm{R}$ had replaced $\theta$ and $\phi$, respectively, as critical letters, and that the critical letter was always in Position 6.

\section{Results}

Verification error proportions for GA, AM, and LF, respectively, were $.00, .00$, and .01 . The correct-detection proportions for each slide type are presented for each $S$ in Table 4. For each $S, z$ tests were computed to compare each slide type having a rectangle with the control slide type. For all Ss the postrectangle-zero comparison was significant at far beyond the .001 level. For SGA the postrectangle-one comparison was significant at the .05 level but the prerectangle comparisons were not; for AM the postrectangle-zero comparison was the only comparison significant at the .05 level; for LF all the comparisons with the control slide type were significant at the .05 level. Two-tailed $\mathrm{z}$ tests were also computed for each $\mathrm{S}$ to compare the prerectangle-zero slide type with the postrectangle-zero slide type. These comparisons were all significant at the .001 level. 


\section{Discussion}

On the basis of the great superiority of the postrectangle-zero slides over the control slides, a masking explanation of the sort considered above must be rejected.

Constraints on further theorizing are the results that the postrectangle-zero effect is in the same direction and of about the same size as the postblank-zero effect found in Experiment 1 for Position 6 with $B$ and $R$ as the critical letters. The significant prerectangle and postrectangle-one effects found for two Ss were small relative to the postrectangle-zero effect and did not show a consistent pattern over Ss. Thus, the effect of a rectangle in any position seems similar to the effect of a blank.

\section{Experiment 5}

Experiment 5 was intended to determine whether the asymmetrical blank effect and the monotonic decrease of detection performance with position of the signal in the instructed processing order could be interpreted as special results of the left-to-right instructed order of the previous experiments. The design was essentially a replication of Experiment 1 except that the focusing dot was placed at the right rather than the left end of the display and the $S$ was instructed to process from right to left.

\section{Subjects}

Two of the Ss were the same as in Experiments 1-4, A Stanford graduate student replaced AM.

\section{Stimulus Materials}

The stimuli were Slide Types $7,8,9,11,14$, and 15 of Table 1 except that $\theta$ and $\phi$ were the critical letters and the background Hs were changed to rectangularly shaped As, i.e., the slide types were the control slides for Positions 5 and 6 , and the slide types with a blank space adjacent to a critical letter in Position 5 or 6 .

\section{Procedure}

The 240 trials per session and the two sessions per day had all the same characteristics as the previous experiments except that the focusing dot appeared at a position at the between-letters distance to the right of the point where the rightmost letter of a display would appear. The Ss were told to begin each trial with their eyes on the focusing dot, to read from right to left, to be nearly always correct on the verification of the rightmost letter, to expect the critical letters $\theta$ and $\phi$ only in the fifth and sixth positions from the right end, to push the $B$ button of Experiment 4 when they detected or guessed $\theta$, and to push the $\mathrm{R}$ button when they detected or guessed $\phi$.

$A$ new $S$ replaced $A M$ and was given a preliminary five sessions of practice. On the basis of this practice, a data-collection stimulus duration of $10 \mathrm{msec}$ was chosen; although this was the shortest duration such that his first-letter verifications were nearly always right, his detection performance on all slides was still above the .55-.60 level desired for Control Position 6 slides. There were two practice sessions and eight data-collection sessions.

\section{Results}

Verification error percentages were $.00, .02$, and .04 for Ss GA, $B M$, and LF, respectively. The correct-detection proportions for each slide type are shown by $S$ in Table 5 . All z-test comparisons of postblank-zero space slides (blank space immediately to the left of the signal) with control slides were significant at the .001 level. No comparison of a preblank-zero proportion with the control proportion of the same position was significant at the.05 level except for the Position 5 comparison for S LF.

\section{Discussion}

It seems that the results of the previous experiments are not
Table 5

Correct Detection Proportions

\begin{tabular}{|c|c|c|c|}
\hline & GA & $\mathbf{B M}$ & LF \\
\hline $\begin{array}{l}\text { Position five: } \\
\text { Postblank zero } \\
\text { Preblank zero } \\
\text { Control }\end{array}$ & $\begin{array}{l}.942 \\
.588 \\
.576\end{array}$ & $\begin{array}{l}.973 \\
.910 \\
.916\end{array}$ & $\begin{array}{l}.948 \\
.865 \\
.734\end{array}$ \\
\hline $\begin{array}{l}\text { Position six: } \\
\text { Postblank zero } \\
\text { Preblank zero } \\
\text { Control } \\
\end{array}$ & $\begin{array}{l}.914 \\
.508 \\
.560 \\
\end{array}$ & $\begin{array}{l}.957 \\
.774 \\
.803 \\
\end{array}$ & $\begin{array}{l}.837 \\
.688 \\
.629\end{array}$ \\
\hline
\end{tabular}

restricted to the left-to-right processing order and, thus, that the reading experience of the Ss was not an important variable in determining the effect of a blank space on detection.

\section{DISCUSSION}

A monotonic decrease in detection probability with distance of the signal from the focusing dot was found in Experiments 1 and 5. Detection performance was approximately the same when the space in the display between the focusing dot and the signal was entirely filled with letters as when a sizable part of this space was blank or contained a black rectangle. These results suggest that a brief, two-dimensional display is processed serially with the rate of processing along a given processing path being independent of the visual content within the path.

The striking feature of the present experiments was the great facilitation of detection performance produced by a blank space or a black rectangle that came immediately after the signal in the processing order. This result seems to place a strong constraint on possible theories that would account for both the obtained spatial detection gradient and the asymmetrical pattern of facilitation. An explanation assuming parallel processing with different processing rates for different locations, for example, does not predict this pattern of facilitation without arbitrary additional assumptions. That both a blank space and a black rectangle can greatly facilitate detection performance and that this large facilitation occurs only when the space or rectangle occurs immediately after the signal do not appear to be predictable from an interpretation in terms of simultaneous masking.

The results of Experiments 3 and 5 limit theoretical alternatives in other ways. Experiment 3 tested the explanation that the left-right asymmetry of the critical letters used in Experiment 1 might have produced the asymmetrical pattern of blank-space facilitation. With symmetrical critical letters in Experiment 3, the same asymmetrical pattern of Experiment 1 was found. Experiment 3 also weighs heavily against the hypothesis that a voluntary processing jump to the signal would produce an effect as large as the facilitation of a postblank-zero space. In Experiment 3 foreknowledge of the position of the signal did not greatly improve the general level of detection performance or change the asymmetrical pattern of facilitation from blank spaces.

Experiment 5 indicates that the critical factor in producing the asymmetrical facilitation was the relationship of the position of the blank space to the position of the signal in the processing order, not simply the left-right relationship of the blank space and the signal in the visual field. In this experiment the instructed processing order was changed to a right-to-left order. A blank space immediately to the left of the signal was immediately after the signal in the processing order and produced the same postblank-zero facilitation found in earlier experiments with a left-to-right order.

The regularity of the preblank-zero, postblank-zero, and control curves of the present Experiment 1 and the repeatability of the major features of these curves from $S$ to $S$ encourage one to attempt a quantitative account of these results. Two critical 
ingredients in this enterprise are the specification of the form of the initial visual representation in the nervous system and a mathematical description of the decay of this representation.

Results directly relevant to the question of the form of the representation and its decay come from a preliminary modified visual detection experiment. The control slides were similar to the control slides of Experiment 1 except that the noise letters were chosen from a set of nine consonants rather than a set of two. 6 The special feature of this experiment was that each experimental slide contained two duplicate critical letters. The result of interest was that detection performance on experimental slides with a critical letter in Position $n$ and a duplicate critical letter in Position $n+i$ was higher than on control slides with a single critical letter in Position n.

The serial processing model of Estes and Taylor (1964) assumes that the entire representation of the display passes below a threshold as a unit and that before this time letters are processed at a constant rate, i.e., all the letters in the initial representation in the nervous system effectively disappear together. But if letters are perfectly processed in the instructed order and the entire representation of the display disappears as a unit, then a second duplicate critical letter later in the processing order cannot aid detection performance. If the display representation disappears after the first critical letter is processed, then the second signal is unnecessary and cannot help; if the display representation disappears before the first signal is processed, then neither signal is processed and the second signal cannot help. Thus, the results of this preliminary experiment suggest that the representation of the display does not disappear as a unit.

It is unnecessary, however, to assume that the entire representation of a display, or even of a letter, disappears as a unit. The critical letters $B$ and $R$ can be distinguished on the basis of much less than the entire detail of each letter. Any dark spot remaining along the bottom of the signal representation indicates that the signal was a " $B$ " rather than an "R." For a given set of critical letters and for a given $S$ some relatively small number of subareas of the signal image might be adequate for differentiation of all letters in the set. These subareas will be referred to henceforth as "critical features." In the simplest case, processing of any one of the features would be independent of processing of any other feature.

In the present experiments the signal letters were quite similar and had curved components; none of the noise letters had any curved components. Because it was much harder to discriminate one signal from the other than to discriminate a signal letter from a noise letter, it seems possible to neglect the process of discriminating a signal image from a noise image in an account of detection performance in the present experiments.

There are numerous grounds for assuming that the amount of information that can be extracted from a critical feature must depend jointly on the clarity of the feature at the time of processing and on the duration of processing spent on the feature. An increase in processing time on the feature can compensate to some degree for a given amount of decay of the feature. Further, it seems essential to assume that on the average the clarity of a feature decreases as a function of the time following stimulus exposure. For simplicity, in the model to be presented, it is conceived that the loss of clarity that makes the feature useless for discrimination of the signal letters (under given conditions of processing duration) is a discrete event, denoted a "fatal decay event." It is also assumed that, to any set of experimental conditions that determines a particular duration of processing time per feature, there corresponds a unique value of the parameter representing the probability of the occurrence of a fatal decay event during a unit time interval.

Specific assumptions of a model for the results of Experiment 1, embodying these notions of the form of the signal image and the nature of the decay process, are as follows:

(1) A signal image has some number n (a small integer, possibly equal to one) of critical features.

(2) Fatal decay events for a critical feature of the image of a given signal letter occur randomly at a mean rate $\lambda$ per unit time, where $\lambda$ is a nonnegative constant.

(3) Processing of a critical feature of a signal $\mathrm{k}$ letter units of distance from the focusing dot begins at time $t_{k}=k t_{1}$, where $t_{1}$ is a positive constant.

(4) Fatal decay events for different critical features of a signal image are independent events.

(5) A critical feature is successfully processed if it has undergone no fatal decay event before its processing is initiated.

(6) A signal is correctly detected if any critical feature of its image is successfully processed.

(7) The value of the parameter $\lambda$, for a given set of experimental conditions, is an increasing function of the processing time available for the critical letter. This duration is independent of the distance of the critical letter from the focusing dot and of the positions of noise letters not adjacent to the critical letter.

These assumptions lead immediately to a simple expression for the probability of detection of a critical letter in any position, $k$ letter units from the focusing dot. The additional notation required is the following: $f_{k}$, the probability that a critical feature of a signal in Position $k$ is successfully processed; $r_{k}$, the probability that a signal in Position $k$ is correctly detected; $P_{k}$, the probability that a correct detection response is made to a slide with a signal in the $\mathrm{k}^{\text {th }}$ position. For a critical feature of a signal in the $\mathbf{k}^{\text {th }}$ position of a slide, it follows that

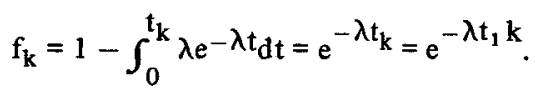

By Assumption 6, $\mathrm{r}_{\mathrm{k}}$ equals the probability that not all critical features of a signal in Position $k$ failed to be processed, i.e.,

$$
r_{k}=1-\left(1-f_{k}\right)^{n}=1-\left(1-e^{\lambda t_{1} k}\right) n
$$

Since the two critical letters occur equally often in a random order, and since $\mathbf{P}_{\mathbf{k}}$ must equal the probability that the signal in Position $k$ is either correctly detected or that the signal is not correctly detected but is correctly guessed,

$$
P_{k}=I_{k}+\left(1-r_{k}\right) \frac{1}{2}=1-\frac{\left(1-e^{-\lambda t_{1} k}\right)^{n}}{2} .
$$

To apply this result to Experiment 1, we need only estimate the product $\lambda t_{1}=m$ separately for the two principle slide types to be compared-the control slides and the postblank-zero slides-together with a common value of $n$, since the latter should be constant over conditions. For each pair of values of $n$ and $m$, the chi-square statistic was used to estimate the goodness of fit of the values of $P_{k}$ to the observed correct-detection proportions for control slides of Experiment 1. The values of $\mathrm{n}$ searched were 1 , $2, \cdots, 17$; the values of $m$ searched were $.01, .02, \cdots, 2.00$. The values of $P_{k}$ which minimized $\chi^{2}$ and the corresponding observed control proportions are presented separately for each $S$ in Fig. 4. The pairs of $n$ and $m$ that minimized $\chi^{2}$ for each $S$ were $(16, .95)$, $(5, .37)$, and $(8, .56)$ for Ss GA, AM, and LF, respectively. These three values of $n$ were then used to find values of $m$ that minimized the $\chi^{2}$ for the fit of new values of $P_{k}$ to the postblank-zero proportions for each $S$ of Experiment 1. These curves are also presented in Fig. 4. The new values of $m$ produced by this procedure were $.58, .26$, and .30 for Ss GA, AM, and LF, respectively. The minimum values of $\chi^{2}$ for the controlproportion fits were $2.0,27.2$, and 6.0 for Ss GA, AM, and LF, 

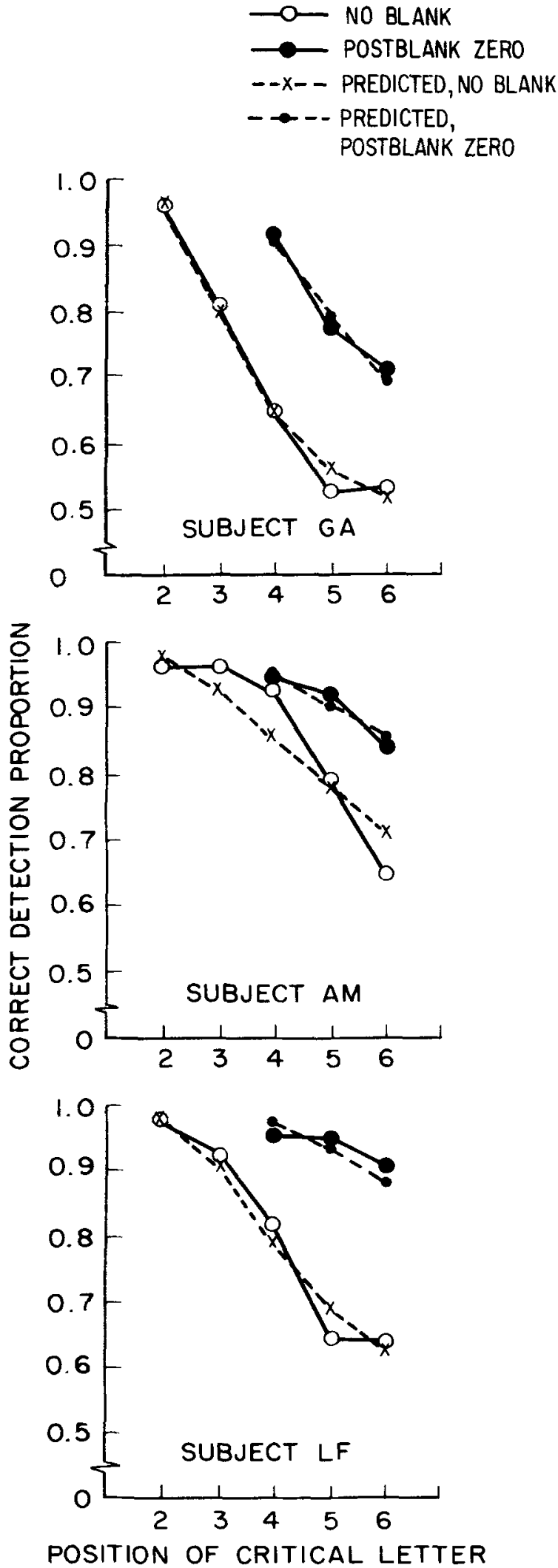

Fig. 4, Observed and theoretical correct-detection proportions as a function of position of the critical letter for Ss GA, AM, and LF. respectively. The minimum values of $\chi^{2}$ for the postblank-zero proportions were 1.7, 1.7, and 5.7 for Ss GA, AM, and LF, respectively.

In summary, a model assuming serial processing of a rapidly decaying representation of the display seems to provide an adequate account of the spatial detection gradient of the present experiments. Still remaining is the task of spelling out the properties of a serial processing mechanism that could generate the pattern of effects produced in the present experiments by a blank space or a rectangle inserted in the S's processing order at various positions relative to the signal.

A promising assumption is that the average duration of the processing of the decayed signal representation is determined by the extent of the area in the processing path following the signal but preceding the next letter. With this assumption the blank space and the rectangle immediately following the signal have the same, positive effect because they take up the same area and cause the decayed signal trace to be processed for the same, extra-long duration. If processing is serial, a blank space or a rectangle preceding the signal has already been shown to delay the processing of a signal trace for approximately the same duration as does the same area filled with letters. A blank space or a rectangle, therefore, takes up some kind of processing time; but if this line of reasoning is correct, a blank space or rectangle does not take up letter processing time.

A serial processing model specifying extra processing time for a letter followed by a blank space or rectangle was the principal alternative to a masking hypothesis that was considered in the design of Experiment 4. This model assumes that the processing of the representation of a visual display involves a character finder and a character reader. The finder processes the area within a processing path at a constant rate, which is independent of the number and nature of the visual objects within the path. In the control slides of the present experiments the finder, therefore, locates letters in the instructed processing order at a constant rate.

The character reader processes only one character at a time and has no waiting line. The reader begins processing each character when the finder inputs the character to the reader; the reader stops processing a character when the finder inputs the next character. The time the reader devotes to the $i^{\text {th }}$ character in the processing order depends, therefore, on the time taken by the finder to locate the $(i+1)^{\text {th }}$ character. This time in turn depends on the distance in the display along the processing path between the $i^{\text {th }}$ character and the $(i+1)^{\text {th }}$ character. With respect to the results of the present experiments, the critical function of the reader is to read letters out of the initial visual image before this trace has decayed too much. It is assumed that any increase in the processing time on a decayed letter trace yields an increase in the amount of information about that letter that is read out of the initial representation and preserved long enough for a motor response.

To identify an area of the display as a character, the finder must be able to distinguish some global properties of visual objects. Experiment 4 was planned with the expectation that a long, black rectangle, with its simple outline and a size greater than that of three letters, would not be identified by the finder as a character and, therefore, that a rectangle immediately following the signal would delay the finding of the next character and would produce the same facilitation of signal detection as a blank space of the same size in the same position. Since the speed with which a character is located depends only on how much area must be processed by the finder before the signal is found, a rectangle before the signal would have the same effect as a blank space of the same area or as the same area filled with letters. This conclusion follows whether or not the finder identifies the rectangle as a character. The character finder seems to be an 
example of the "preattentive processes" advocated in a general way by Neisser (1967). Neisser gives several interesting, but complex, arguments for "wholistic operations which form the units to which attention may then be directed."

The finder-reader serial processing model gives a good qualitative account of the main effects of the present experiments. It gives a simple explanation of why either a blank space or a rectangle coming immediately after the signal in the processing order produces a positive effect of the same magnitude on detection performance; it prescribes that slides with a blank space or a rectangle coming before the signal and control slides with a signal in the same position should show equal detection performance.

\section{REFERENCES}

ESTES, W. K., \& TAYLOR, H. A. A detection method and probabilistic models for assessing information processing from brief visual displays. Proceedings of the National Academy of Sciences, 1964, 52, No.2, 446-454.

ESTES, W. K., \& TAYLOR, H. A. Visual detection in relation to display size and redundancy of critical elements. Perception \& Psychophysics, $1966,1,9-16$.

ESTES, W. K., \& WESSEL, D. L. Reaction time in relation to display size and correctness of response in forced-choice visual signal detection. Perception \& Psychophysics, 1966, 1, 369-373.

FERGUSON, G. A. Statistical analysis in psychology and education. McGraw-Hill, 1959.

NEISSER, U. Cognitive psychology. New York: Appleton-Century-Crofts, 1967.
SPERLING, G. The information available in brief visual presentations. Psychological Monographs, 1960, 74, No. 11 (Whole No. 498).

SPERLING, G. A model for visual memory tasks. Human Factors, 1963, 5 , 19-32.

SPERLING, G. Successive approximations to a model for short term memory. Acta Psychologica, 1967, 27, 285-292.

\section{NOTES}

1. This report is based on a dissertation submitted in partial fulfillment of the requirements for the PhD degree at Stanford University. I am indebted to $J o h n$ E. Holmgren for extensive help in programming and modifying the apparatus and to Stuart Miller for several data-analysis computer programs. I am especially grateful to Dr. William K. Estes for his encouragement and invaluable advice. The research reported here was done while the author held a predoctoral fellowship from the National Institute of Mental Health.

2. Address: The Rockefeller University, New York, N. Y. 10021

3. Now at The Rockefeller University.

4. Originally all background letters were $H$ or $N$ but preliminary trials showed that $\mathrm{H}$ and $\mathrm{N}$ were not well discriminated at short exposure durations. To improve the verification discrimination the initial $\mathrm{Hs}$ in the arrays were changed to rectangularly shaped As.

5. Each $S$ reported that motor mistakes were rare after the practice sessions.

6. An incidental result of this experiment was an indication that selection of the noise letters from a larger set did not produce a different level of detection performance. SGA was in this experiment and Experiment 1, and his performance on control slides in the two experiments was quite similar.

(Accepted for publication March 20, 1969.) 